\title{
A Fast and Precise HOG-Adaboost Based Visual Support System Capable to Recognize Pedestrian and Estimate Their Distance
}

\author{
Kishino Takahisa, Zhe Sun, and Ruggero Micheletto \\ Yokohama City University, Graduate School of Nanobioscience, \\ 22-2 Seto Kanazawa-ku, 236-0027 Yokohama, Japan \\ \{n115208c,n125213e, ruggero\}@yokohama-cu.ac.jp \\ http://ruggero.sci.yokohama-cu.ac.jp/
}

\begin{abstract}
In this paper,we present a visual support system the visually impaired. Our detection algorithm is based on the well known Histograms of Oriented Gradients (HOG) method, due to its high detection rate and versatility 5. However, the accuracy of object recognition rate is reduced because of high false detection rate. In order to solve that, multiple parts model and triple phase detection have been implemented. These additional filtering stages were conducted by separate action on different area of the sample, considering deformations and translations. We demonstrated that this approach has raised the accuracy and speed of calculation. Through an evaluation experiment based on a large dataset, we found that false detection has been improved by $18.9 \%$ in respect to standard HOG detectors. Experimental tests have also shown the system ability to estimate the distance of the pedestrian by the use of a simple perspective model. The system has been tested on several photographic datasets and have shown excellent performances also in ambiguous cases.
\end{abstract}

Keywords: Pedestrian detection, HOG methods, distance evaluation, single-camera, Adaboost.

\section{Introduction}

In this study, we present steps toward the realization of an automatic pedestrian detection system for the visually impaired. Today, the visually impaired has many obstacles to walk outside and despite the advancement of technology in many fields, we often see blind people accompanied by friends or by a dog. Widespread devices to help these persons are not common yet. However, we think that audio support devices that are based on a GPS route navigation systems 8 are promising and may be successful. And they also present dangers because of possible collision by other pedestrian.

Many of the pedestrian detecting systems typically apply standard computer vision techniques 11, such as background subtraction or background modelling [10]. Since the camera is given to the visually impaired, the images are shot directly from the user viewpoint. As an alternative for background subtraction, it is possible to 
use the approach of detecting multiple and deformable parts. This approach will be used in this study and its results shown. We will demonstrate that it is possible to reduce the effect of a variable and dynamic background and obtain high quality detection and robust results.

Our system consists of an algorithm that acts in two phases: pedestrian detection and estimation of distance. In the detection phase, it scans the image for the pedestrian shape standing in front of the camera. We improved a standard HOG method introducing a multiple part model to it.

\subsection{Comparison of HOG, SIFT and PCA-SIFT}

We realized that HOG method is more suitable than other methods because of its geometrical and optical transfer invariance, and because it shows low computational complexity and high velocity [9].

Table 1. A rough comparison between HOG, SIFT and PCA-SIFT methods for pedestrian recognition

\begin{tabular}{|c|c|c|c|}
\hline & Speed & Scale & Rotation \\
\hline HOG & best & best & common \\
SIFT & common & good & $\begin{array}{c}\text { good } \\
\text { best }\end{array}$ \\
PCA-SIFT & good & common & Pemol \\
\hline
\end{tabular}

\section{System Model}

This section describes the pedestrian detection system and the distance estimation process.

\subsection{Histograms of Oriented Gradients}

The underlying building blocks of our method are the Histograms of Oriented Gradients (HOG) 4. HOG representation captures the gradient structure that is characteristic of the human shape. A magnitude $m$ and orientation $\theta$ of gradients at each pixel are given by the equation:

$$
\begin{array}{r}
m(x, y)=\sqrt{f_{x}(x, y)^{2}+f_{y}(x, y)^{2}} \\
\theta(x, y)=\tan ^{-1} \frac{f_{x}(x, y)}{f_{y}(x, y)}
\end{array}
$$

where $f_{x}(x, y)=L(x+1, y)-L(x-1, y), f_{y}(x, y)=L(x, y+1)-L(x, y-1)$ and $L(x, y)$ is proportional to the brightness of a pixel. $\theta$ is discretized into one of nine orientation bins. Each pixel is assigned the orientation of its gradient, with a strength that depends on $m$. The image is divided into $n * n$ 


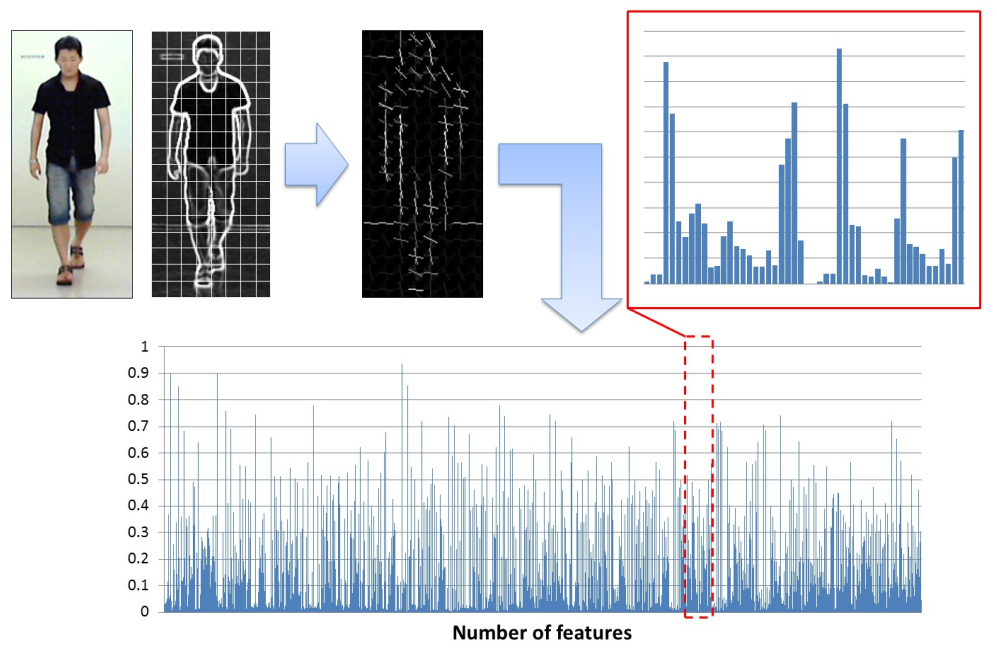

Fig. 1. An example of the HOG process. The HOG analysis acts on cells that contain the images edges of the image captured by the camera. The edges are evaluated for orientation $\theta$ and an orientation magnitude $m$ value is calculated for each cell and normalized. The magnitudes $m$ are normalized by blocks (see text for details). These normalized magnitudes are stored as an one-dimensional histogram and represent the main feature of the image that will be compared with a database of positive or negative samples for the human recognition process.

not overlapping pixel regions that are called cells and each group of cells is integrated into a block. The blocks can overlap with each other. These gradient features are represented as one-dimensional histogram, as shown in Figure 1 .

\subsection{Multiple Parts Model}

The pedestrian have diverse postures (e.g. looking down, checking the phone). From the point of view of the camera held by the subject, the relative changes in the parts (e.g. head, arm) position are especially important. So our system is required to deal with various poses without making mistakes. One of the methods to detect diverse human poses, consists of divide the target object into several parts [6] and consider the whole as the composition of them. Thus our method treats the person model as a cluster consisting of three parts, that define the complete body, the head and the legs, see Figure. 2. The model of the pedestrian is composed by a base filter and two secondary models $\left(P_{1}, P_{2}\right)$. The base filter $F_{0}$ covers the entire human and defines the rough pedestrian location. The secondary models represent the head and feet parts. In general each secondary model is given by the relation:

$$
P_{i}=\left(F_{i}, v_{i}, s_{i}\right)
$$




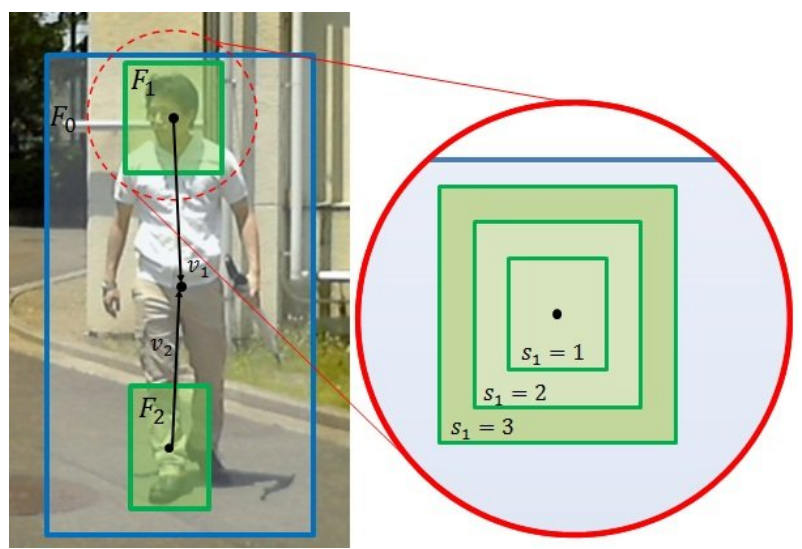

Fig. 2. A sketch representing how the multiple part analysis is done. We apply the HOG algorithm firstly on the whole screen and by the use of a mean-shift clustering procedure the position of the person body is located. To realize a more robust analysis we apply the HOG algorithm again twice in order to locate the head and the feet parts of the image. This further analysis is made using the three different sizes $s_{i}$ as mentioned in the text to reduce error.

Here $i$ is the part number, $F_{i}$ is the part filter for the specific $i$ th element, $v_{i}$ is a two dimensional vector locating the center of a box enclosing the part and finally $s_{i}$ gives the size of the box. There are three sizes for each box to reduce error.

We have considered that using higher resolution is essential to reduce false detections. Therefore each part filter $f_{i}$ has a higher HOG resolution than its base. The position of each filter can move at each scene, remaining confined within its base filter range. The filter can act externally to the base filter provided it is overlapping with it at least $50 \%$ of its area. In this way the variability is enough to recognize the region of the body in diverse position without incurring in errors.

\subsection{Classifier Construction}

To optimize performances we used a cascade AdaBoost classifier 13 14 trained by the main HOG features 12 . When the processing is complete, the final classifier $H(x)$ is a linear combination of several weak classifiers $h_{t}(x)$. The number of weak classifiers is $T$ and it is equal in number as the learning samples.

$$
H(x)=\sum_{t=1}^{T} \alpha_{t} h_{t}(x)
$$

where $x$ is the input feature data, $t$ is number of learning round. $\alpha_{t}$ is the weight of the $t$ th learning data, this it is given by the equation: 


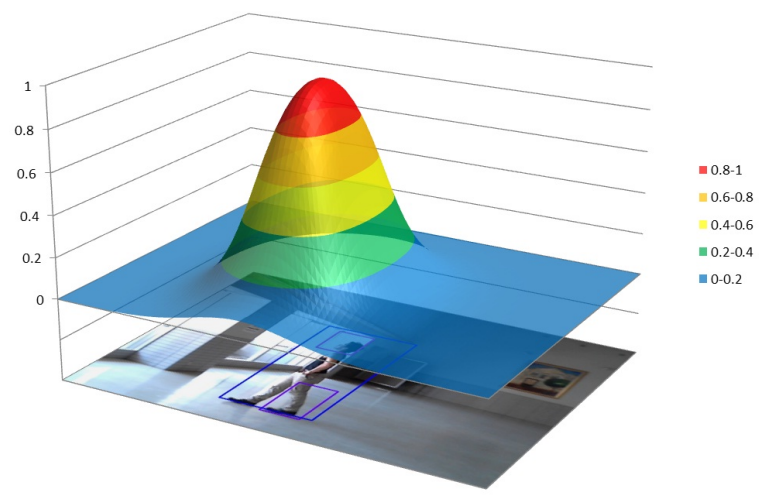

Fig. 3. An example of the likelihood distribution of a image. For each image frame captured by the camera, a likelihood map is generated in real time. This map is obtained from output values of the classifiers that scans the image in an iterative process. When a region of the image is found to have similar features to the human profile accordingly to a database of learning data, that area is assigned a higher likelihood.

$$
\alpha_{t}=\frac{1}{2} \ln \frac{1-e_{t}}{e_{t}}
$$

where $e_{t}$ is the weight summation of each learning sample at th learning round.

In other words, the output value of the final classifier represents the level of resemblance to human profile of a particular area on the image. When the classifier is applied to the image area where a human is present, it outputs a high value. By iteration of raster scans, a map that associates the human likelihood to the image is generated. As shown in Figure 3, our classifier likelihood is mainly distributed over the human profile in the example.

The features of HOG are compared with positive samples (human images) and negative ones (background object and other non-human images). The features that exhibit greater differences between positive and negative samples are considered for an efficient classifier, about 200 classifiers were selected in this study.

We also compared the AdaBoost classifier algorithm with the SVM classifier and we realized that a weak classifier AdaBoost with high-speed detection is more suitable for pedestrian recognition [7.

\subsection{Distance Range Estimation}

To notify the user of collision risks, our system estimate the distance to pedestrian that are located ahead the camera 3. As the image is shot from a single camera the distance should be estimated by perspective projection. There are two clues to estimate distance by perspective: the size of pedestrian in the image 
and its position. Since the size of pedestrian depends on the person body height, our system derives the approximate range using the position of the pedestrian. We assume a planar road surface and that a camera optical axis is parallel to the road surface, even though the system is robust enough to accept small perturbations around these conditions ( see Figure 4 with a diagram of the imaging geometry).

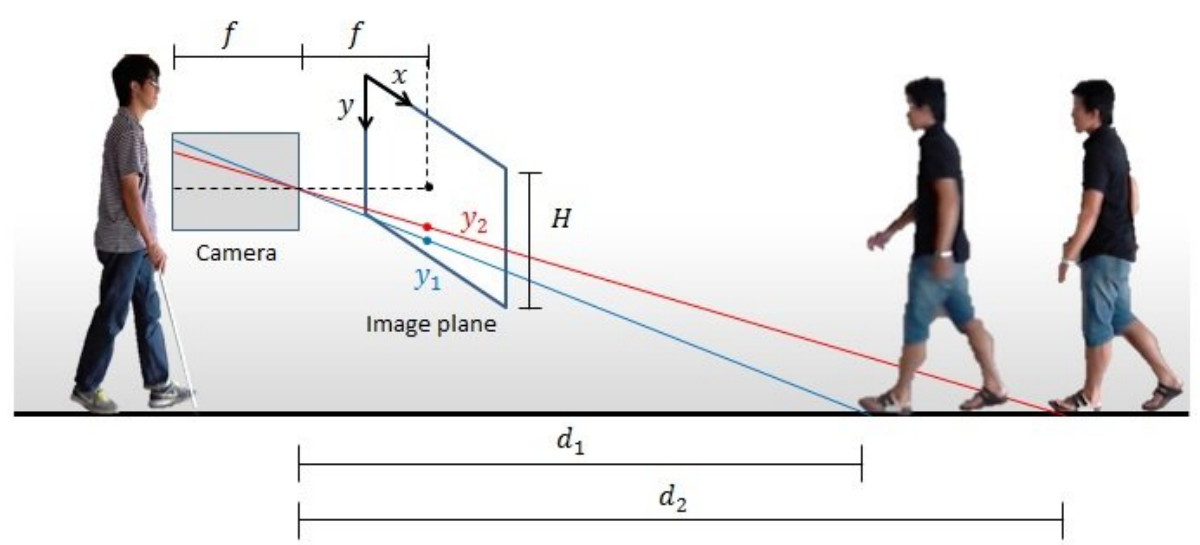

Fig. 4. Ideal image geometry in the perspective model for distance estimation. See text for details.

The camera is held by the user at height $h$. The distance to the pedestrian from the camera is $d$. The point on the road at the distance $d$ is projected onto the image plane at the position $y$. This is the image coordinates given by the equation:

$$
y=\frac{f h}{d}+\frac{H}{2}
$$

where $f$ is the camera focal length expressed in pixel and $H$ is the full screen size.

When a first pedestrian is at a distance $d_{1}$ and a distant pedestrian is at $d_{2}$, the points of contact are projected onto the image at $y_{1}$ and $y_{2}$. As shown in the example of Figure. 4, $y_{1}$ results to be smaller than $y_{2}$. To calculate the distance to a pedestrian the system detects the person foot and its range is estimated from the following formula:

$$
d=\frac{1}{f h}\left(y-\frac{H}{2}\right)
$$

In the current algorithm the camera is set at $h=1.4 \mathrm{~m}$ and as an indication to notify of the collision risk, pedestrian are grouped by their distance into three range levels: near-range (4m or less), medium-range (between $4 \mathrm{~m}$ and $8 \mathrm{~m}$ ), and 


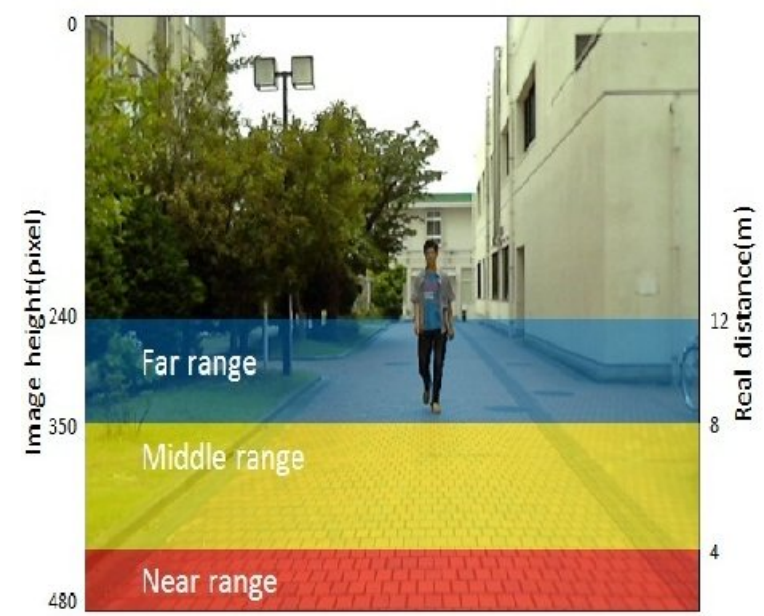

Fig. 5. The relation with distance shown on the camera image

far-range (8m or more). Figure 5 shows this distant relationship superimposed to the camera image.

\section{Experiment and Results}

\subsection{Experiment}

To evaluate detection accuracy, we prepared a dataset.Using this dataset we compare two methods of detection, conventional HOG and our multi parts detector. Table 2 shows the accuracy result.

Even if the conventional HOG detector has a better detection rate than our method, the difference in accuracy is just $3.2 \%$. On the contrary, compared to the conventional HOG method, our method have a $18.92 \%$ better false detection performance. Since our method have a flexible positioning of each part the decrease in detection rate is low. Instead the false detection result, the conventional HOG method is more likely to falsely detect objects with a complex texture. Overall, our method makes a slight sacrifice in detection rate, to obtain an improved lower false detection rate.

\subsection{Pedestrian Detection}

The process of detecting the pedestrian from an image consists of a detection window that scans the image over and over. The scale and position of this window are changed in scale and position in an optimized way. In this way it is possible to detect humans whose size is diverse. These images are taken in multiple locations, with a resolution of $640 * 480$ pixels. 
Table 2. Accuracy rate for HOG and our multiple model method. The "miss" parameter shows the percentage of missed target, while "false" include the missed targets and the detection of false targets.

\begin{tabular}{|c|c|c|c|}
\hline & precision & miss & false \\
\hline HOG & 91.90 & 9.10 & 25.21 \\
Multiple Model & 88.70 & 11.30 & 6.29 \\
\hline
\end{tabular}

(a) Conventional HOG method
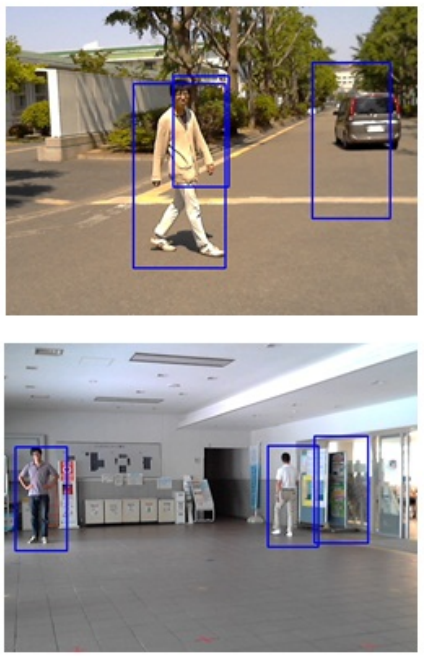

(b) Multiple parts HOG method
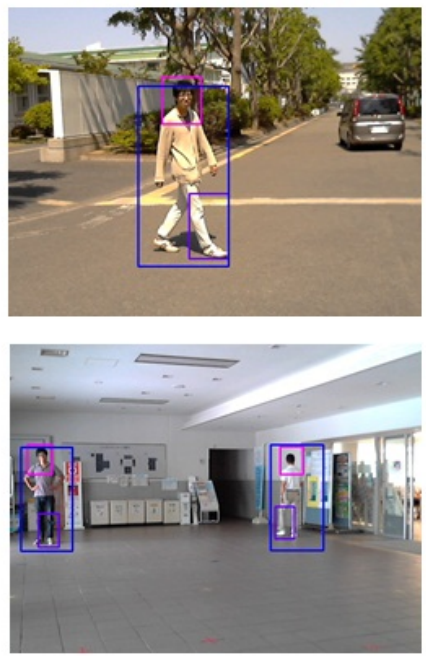

Fig. 6. Examples of detection images over complex and moving backgrounds. On the left are shown the results with a conventional HOG approach: moving car and other objects are falsely detected. On the right our multiple part HOG approach solves this problem when tested on the same video frames.

In Figure 6 we show a comparative result of pedestrian detection examples. The conventional method shows false detections due to complex background. Because our method operates with three phase detection, it works better and is more robust than the conventional HOG method. Our method is able to detect pedestrian accurately in these non-ideal environments. Figure. 7 shows the result of estimating the distance of each person. We see that the position of each pedestrian can be estimated with good approximation. Based on this result, our system can compute the collision risk and notify the user of approaching pedestrian.

\section{Conclusions and Perspective}

In this study, we proposed a pedestrian detection system which can estimate the distance to targets with a single camera by the use of a multiple-parts HOG 

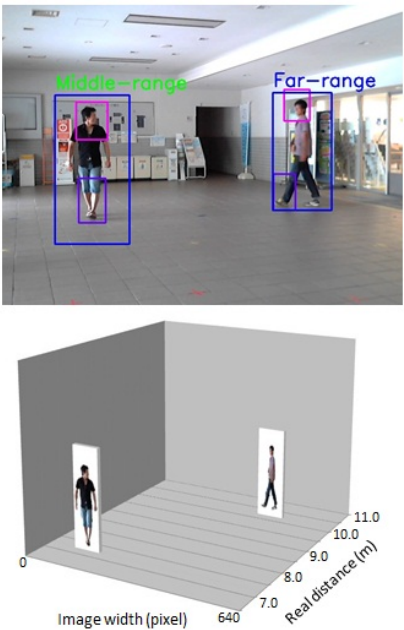
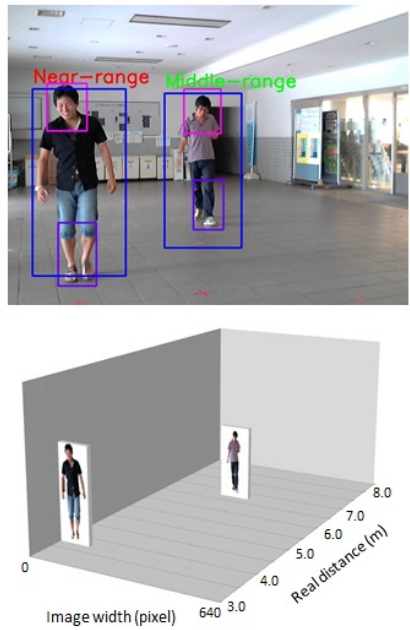

Fig. 7. Examples of detection images and the corresponding distance estimation range

model. The result of test experiments shows that our detection method has a $18.92 \%$ improvement in false detection rate against the conventional HOG method. The system was also able to detect pedestrian in complicated backgrounds moving environments. The system was able to estimate the distance of pedestrian using the single camera. It is possible to derive collision risk from the estimated distance. Our system can be implemented on simple and convenient devices (e.g., smartphones and tablet computers). We are planning to improve the system developed up to now to work as a support application in real environments. Especially there we want to focus on two parameters, real time processing and pedestrian tracking. Our system takes more CPU time than the simple HOG method. We have to optimize processing time and simultaneously realize the tracking of the pedestrian to support the user to decide the direction of avoidance. We plan to use time-series filtering, Kalman filter [2] or Particle filter [1]. By applying a time-series filter algorithm, faster process time is expected because of the determinate scan area. The new filtering will also enable to improve the accuracy because of the tracking information relative to the target person.

\section{References}

1. Brasnett, P., Mihaylova, L., Bull, D., Canagarajah, N.: Sequential monte carlo tracking by fusing multiple cues in video sequences. Image vision Computing 25(8), 1217-1227 (2007)

2. Cuevas, E., Zaldiver, D., Rojas, R.: Kalman filter for vision tracking. Fachbereich Mathematik und Informatic. Technical Report B, Freie Universitat Berlin (2005)

3. Hoiem, D., Efros, A.A., Hebert, M.: Putting objects in perspective. Pattern Analysis and Machine Intelligence 2 (2006) 
4. Dalal, N., Triggs, B.: Histograms of oriented gradients for human detection. In: CVPR, pp. 886-893 (2005)

5. Suard, F., Rakotomamonjy, A., Bensrhair, A., Broggi, A.: Pedestrian detection using infrared images and histograms of oriented gradients. In: Proc. IEEE Conf. Intell. Vehicles, pp. 206-212 (2006)

6. Felzenszwalb, P., Girschick, R., McAllester, D.: Cascade object detection with deformable part models. In: CVPR, pp. 1-8 (2010)

7. Han, F., Shan, Y., Cekander, R., Sawhney, H.S., Kumar, R.: A two-stage approach to people and vehicle detection with hog-based svm. In: Performance Metrics for Intelligent Systems Workshop in conjunction with the IEEE Safety, Security, and Rescue Robotics Conference, pp. 134-136 (2006)

8. Helal, A., Moore, S., Ramachandran, B.: Drishti: An integrated navigation system for the visually impaired and disabled. In: Fifth International Symposium on Wearable Computers (ISWC 2001), pp. 149-156 (2001)

9. Juan, L., Gwun, O.: A comparison of sift, pca-sift and surf. International Journal of Image Processing 3, 143-152 (2009)

10. Montabone, S., Soto, A.: Human detection using a mobile platform and novel features derived from a visual saliency mechanism. Image and Vision Computing 28(3), 391-402 (2010)

11. Felzenszwalb, P.F., Girshick, R.B., McAllester, D., Ramanan, D.: Object detection with discriminatively trained part based models. Pattern Analysis and Machine Intelligence, 1627-1645 (2010)

12. Zhu, Q., Avidan, S., Yeh, M.C., Cheng, K.T.: Fast human detection using a cascade of histograms of oriented gradients. Mitsubishi Electric Research Laboratories, pp. 1491-1498 (2006)

13. Yan, X., Luo, Y.: Recognizing human actions using a new descriptor based on spatial-temporal interest points and weighted-output classifier. Neurocomputing 87, 51-61 (2012)

14. Zhang, T., Liu, S., Xu, C., Lu, H.: Boosted multi-class semi-supervised learning for human action recognition. Pattern Recognition 44(10-11, SI), 2334-2342 (2011) 